\title{
Solutions of Navier-Stokes Equation with Coriolis Force
}

\author{
Sunggeun Lee, ${ }^{1}$ Shin-Kun Ryi, ${ }^{2}$ and Hankwon Lim $^{1}$ \\ ${ }^{1}$ Department of Advanced Materials and Chemical Engineering, Catholic University of Daegu, Gyeongsan, \\ Gyeongbuk 38430, Republic of Korea \\ ${ }^{2}$ Korea Institute of Energy Research, Daejeon 305-343, Republic of Korea \\ Correspondence should be addressed to Shin-Kun Ryi; h2membrane@kier.re.kr and Hankwon Lim; hklim@cu.ac.kr
}

Received 15 April 2017; Accepted 3 July 2017; Published 14 August 2017

Academic Editor: Eugen Radu

Copyright (C) 2017 Sunggeun Lee et al. This is an open access article distributed under the Creative Commons Attribution License, which permits unrestricted use, distribution, and reproduction in any medium, provided the original work is properly cited.

We investigate the Navier-Stokes equation in the presence of Coriolis force in this article. First, the vortex equation with the Coriolis effect is discussed. It turns out that the vorticity can be generated due to a rotation coming from the Coriolis effect, $\Omega$. In both steady state and two-dimensional flow, the vorticity vector $\omega$ gets shifted by the amount of $-2 \Omega$. Second, we consider the specific expression of the velocity vector of the Navier-Stokes equation in two dimensions. For the two-dimensional potential flow $\vec{v}=\vec{\nabla} \phi$, the equation satisfied by $\phi$ is independent of $\Omega$. The remaining Navier-Stokes equation reduces to the nonlinear partial differential equations with respect to the velocity and the corresponding exact solution is obtained. Finally, the steady convective diffusion equation is considered for the concentration $c$ and can be solved with the help of Navier-Stokes equation for two-dimensional potential flow. The convective diffusion equation can be solved in three dimensions with a simple choice of $c$.

\section{Introduction}

Our Earth, even though it is hard to feel in daily life, is rotating. However, if we look around to see what is happening, we realize that this rotation leads to many interesting phenomena. These phenomena are unavoidable as long as we live on Earth. On a macroscopic scale, this effect is substantial: the climate is affected and complicated because the rotation changes the flows of the air heated by the sun. The air has a very complicated motion compared to the one without rotation. Typhoons and their motion are another phenomenon resulting from the effect of the rotation of Earth. Furthermore, their direction of rotation is opposite to each other in the northern and southern hemispheres. The flows of the ocean can be affected by the rotation of Earth as well. Changing the motion of moving objects or fluids such as air and ocean illustrated above is caused by a force. This force on all moving bodies is called Coriolis force. The rotating effect may be seen in outer space such as accretion disks of fast rotating black hole [1] as well. In this case, the relativity and the curved space-time should be counted to understand the effect better. It has been applied commercially in the areas of, for instance, Coriolis flow meter, and the solutions to the flow equations of Navier-Stokes equation are very useful. Hence, the Coriolis effect in the Navier-Stokes equation should be included in these cases. In addition, when the long cylinder carrying flows of water or oil is concerned, the Coriolis effect is not negligible and should be considered in order to construct the cylinder safely. In wide aspects, many chemical reactions of the flowing fluids or gases should be understood with the inclusion of this effect.

In summary, in fact, almost all of the fluids flowing on Earth can be affected, so knowing the exact motion of the fluids is important for applications and problem solving. The flow even involves heat flows of the fluids as well. Our work is just a starting point to find such solutions. As a basic exercise, we investigate the vortex equation and the Navier-Stokes equations under the Coriolis force. Specifically, we assume that the solutions are time independent. In [2], the Coriolis effect on the three-dimensional Navier-Stokes equation was considered. That was the case of compressible fluid while our concern here is the case of an incompressible one.

The present work has the following structure. In Section 2, we construct the vortex equation with a term of Coriolis force. The Coriolis effect can generate the vorticity. Especially for two-dimensional flow, the interesting point of view is 
the fact that the angular velocity coming from Coriolis force comes into play just as a shift of vorticity vector. In Section 3, we try to find the solution to the Navier-Stokes equations with Coriolis force as well. We apply the solutions to convective diffusion for two-dimensional potential flow. The effect of rotation does not appear on the velocity potential and the concentration $c$ is independent of its effect. In three dimensions, we take simplified choice of $c$ and solve the convective diffusion equation. Finally, Section 4 finishes with conclusions and discussions.

\section{Vortex Equation for Two-Dimensional Flow}

In this section, we construct the equation satisfied by the vortex [3] arising from the Navier-Stokes equation. When the fluid motion is viscous, the Euler equation becomes replaced by the Navier-Stokes equation. For incompressible flow, the Navier-Stokes equation in the presence of Coriolis force is given by

$$
\begin{aligned}
\frac{\partial \vec{u}}{\partial t}+\vec{u} & \cdot \vec{\nabla} \vec{u}=-\vec{\nabla} p+\operatorname{Re}^{-1} \nabla^{2} \vec{u}-2 \vec{\Omega} \times \vec{u}, \\
\vec{\nabla} \cdot \vec{u} & =0,
\end{aligned}
$$

where Re is Reynolds number. The last term of the first equation comes from the Coriolis effect with the angular velocity $\vec{\Omega}$. The vorticity vector $\vec{\omega}$ is given by

$$
\vec{\omega}=\vec{\nabla} \times \vec{u} .
$$

Let us apply curl $\nabla \times$ on both sides of the Navier-Stokes equation and use the vector identity

$$
\begin{aligned}
\nabla \times(\vec{\Omega} \times \vec{u})= & \vec{\Omega}(\vec{\nabla} \cdot \vec{u})-\vec{u}(\vec{\nabla} \cdot \vec{\Omega})-(\vec{\Omega} \cdot \vec{\nabla}) \vec{u} \\
& +(\vec{u} \cdot \vec{\nabla}) \vec{\Omega}
\end{aligned}
$$

We will focus on the case of constant $\vec{\Omega}$. Then, we see that the vorticity vector satisfies the following differential equations for incompressible fluid $(\nabla \cdot \vec{u})$ :

$$
\begin{aligned}
\frac{\partial \vec{\omega}}{\partial t}+(\vec{u} \cdot \vec{\nabla}) \vec{\omega}= & (\vec{\omega} \cdot \vec{\nabla}) \vec{u}+\nu \nabla^{2} \vec{\omega} \\
& -2(\vec{\Omega} \cdot \vec{\nabla}) \vec{u} \\
= & {[(\vec{\omega}-2 \vec{\Omega}) \cdot \vec{\nabla}] \vec{u}+\nu \nabla^{2} \vec{\omega} }
\end{aligned}
$$

where we changed the notation of Reynolds number as $v=$ $1 /$ Re.

Note that even if the vorticity vector in (4) is only time dependent, that is, without spatial dependence, the Coriolis effect gives rise to the vorticity. In this case, the vorticity is given by

$$
\omega=-2 \int d t(\vec{\Omega} \cdot \vec{\nabla}) \vec{u}+\omega\left(t_{0}\right)
$$

Moreover, we see that, by solving the equation

$$
(\vec{\omega} \cdot \vec{\nabla}) \vec{u}=-2(\vec{\Omega} \cdot \vec{\nabla}) \vec{u},
$$

the vorticity can be generated even for a constant $\omega$ (for $\omega$ independent of both time and space in (4)). This means that the phenomenon of vortex can arise everywhere on Earth even though the effect of Coriolis on Earth is too small to be seen.

If we define $\vec{\omega}_{\Omega}$ such that

$$
\vec{\omega}_{\Omega}=\vec{\omega}-2 \vec{\Omega}
$$

and consider stationary solution such that $\partial \vec{\omega} / \partial t=0$, then we have the following equation:

$$
\vec{u} \cdot \vec{\nabla} \vec{\omega}_{\Omega}=\left(\vec{\omega}_{\Omega} \cdot \vec{\nabla}\right) \vec{u}+\nu \nabla^{2} \vec{\omega}_{\Omega}
$$

Thus, the angular velocity coming from Coriolis effect only appears as a shift of vorticity vector. Furthermore, if we consider two-dimensional flows such that

$$
\vec{u}=\hat{i} u_{x}+\hat{j} u_{y}
$$

where $\hat{i}$ and $\hat{j}$ are unit vectors on $x$ and $y$, respectively, and the angular velocity $\vec{\Omega}$ is a two-dimensional one, then the above equations are reduced to the following:

$$
\vec{u} \cdot \vec{\nabla} \vec{\omega}_{\Omega}=\nu \nabla^{2} \vec{\omega}_{\Omega}
$$

Note that if $\vec{\Omega}$ has one component, say $\Omega_{3}$, affecting the vorticity vector, say $\omega_{3}$, in two-dimensional flow (we express the velocity vector as $\vec{u}=\widehat{i} u_{1}+\hat{j} u_{2}$ ), hence we can discard vector notation and the equation becomes

$$
\vec{u} \cdot \vec{\nabla} \omega_{\Omega_{3}}=\nu \nabla^{2} \omega_{\Omega_{3}} .
$$

Considering the constant pressure case, the Navier-Stokes equation becomes

$$
(\vec{u} \cdot \vec{\nabla}) \vec{u}=\nu \nabla^{2} \vec{u}
$$

Even though the above equation is reduced to a simplified form, it is still hard to be solved. By restricting two dimensions, we may simplify the equation and have a chance to get a solution. Alternatively, changing the above second-order differential equations into a first-order differential equation like BPS solution $[4,5]$ in field theory is an open and challenging problem.

Solving the Navier-Stokes equation directly is a straightforward way to get a vorticity though the exact solutions are quite restricted. In the next section, we try to solve the steady two-dimensional Navier-Stokes equation. 


\section{Solution of Navier-Stokes Equations and Its Applications}

Let us find the solution of Navier-Stokes equations of which velocities have the following expression [6]:

$$
\begin{aligned}
\vec{u}(x, y, z) & =\left(\frac{\partial \phi(x, y)}{\partial x}, \frac{\partial \phi(x, y)}{\partial y}, v(x, y)\right) \\
& =\left(u_{1}, u_{2}, u_{3}\right),
\end{aligned}
$$

where $\phi(x, y)$ is the velocity potential for the flow in the $(x, y)$ plane and $v(x, y)$ is out-of-plane velocity component. The vorticity vector field is given by

$$
\vec{\omega}=\vec{\nabla} \times \vec{u},
$$

and from the expression in (13) we have the vorticity vector

$$
\vec{\omega}=\hat{i}\left(\frac{\partial v}{\partial y}\right)+\hat{j}\left(-\frac{\partial v}{\partial x}\right)=\left(\frac{\partial v}{\partial y},-\frac{\partial v}{\partial x}, 0\right) .
$$

The contours of $v=$ constant are known as the vortex lines of the flow. The zero vorticity comes out if the third component of the velocity $v$ is equal to zero in which it turns out to be a two-dimensional flow.

In components, after inserting $u_{1}, u_{2}$, and $u_{3}$ into the Navier-Stokes equation and focusing on the time independent velocities such that $\partial u_{1,2,3} / \partial t=0$, we have the following set of equations:

$$
\begin{aligned}
& u_{1} \frac{\partial u_{1}}{\partial x}+u_{2} \frac{\partial u_{1}}{\partial y}+u_{3} \frac{\partial u_{1}}{\partial z} \\
& =-\frac{\partial p}{\partial x}+\nu \nabla^{2} u_{1}-2 \Omega_{2} u_{3}+2 \Omega_{3} u_{2}, \\
& u_{1} \frac{\partial u_{2}}{\partial x}+u_{2} \frac{\partial u_{2}}{\partial y}+u_{3} \frac{\partial u_{2}}{\partial z} \\
& =-\frac{\partial p}{\partial y}+\nu \nabla^{2} u_{2}+2 \Omega_{1} u_{3}-2 \Omega_{3} u_{1}, \\
& u_{1} \frac{\partial u_{3}}{\partial x}+u_{2} \frac{\partial u_{3}}{\partial y}+u_{3} \frac{\partial u_{3}}{\partial z} \\
& =-\frac{\partial p}{\partial z}+\nu \nabla^{2} u_{3}-2 \Omega_{1} u_{2}+2 \Omega_{2} u_{1} .
\end{aligned}
$$

The incompressible conditions give the continuity equation:

$$
\frac{\partial u_{1}}{\partial x}+\frac{\partial u_{2}}{\partial y}+\frac{\partial u_{3}}{\partial z}=0
$$

For the expression of the velocity as in (13) in $(x, y)$ plane, the independence of the third direction $z$ gives the following relation:

$$
\begin{gathered}
\frac{\partial u_{1}}{\partial z}=0 \\
\frac{\partial u_{2}}{\partial z}=0 \\
\frac{\partial v}{\partial z}=0
\end{gathered}
$$

Therefore, the continuity equation can be reexpressed as

$$
\frac{\partial u_{1}}{\partial x}+\frac{\partial u_{2}}{\partial y}=\frac{\partial^{2} \phi}{\partial x^{2}}+\frac{\partial^{2} \phi}{\partial y^{2}}=0 .
$$

Finally, we assume the pressure is also independent of the third direction:

$$
\frac{\partial p}{\partial z}=0
$$

Since the specific choice of the velocities reduces the three-dimensional problems to two-dimensional problems and working in the complex coordinates might have a number of advantages thanks to the numerous available techniques in complex analysis (e.g., conformal mapping), we now introduce the complex coordinates such that

$$
\begin{aligned}
z & =x+i y, \\
\bar{z} & =x-i y, \\
d z & =d x+i d y, \\
d \bar{z} & =d x-i d y .
\end{aligned}
$$

So, by applying chain rule, we get the following relations:

$$
\begin{aligned}
& \frac{\partial}{\partial x}=\frac{\partial}{\partial z}+\frac{\partial}{\partial \bar{z}}, \\
& \frac{\partial}{\partial y}=i \frac{\partial}{\partial z}-i \frac{\partial}{\partial \bar{z}} .
\end{aligned}
$$

Now, let us rewrite the Navier-Stokes equation in complex coordinates by replacing derivatives in real coordinates with complex coordinates:

$$
\begin{aligned}
& 2\left(\frac{\partial}{\partial z}+\frac{\partial}{\partial \bar{z}}\right)\left(\frac{\partial \phi}{\partial z} \frac{\partial \phi}{\partial \bar{z}}\right) \\
& \quad=\left(\frac{\partial}{\partial z}+\frac{\partial}{\partial \bar{z}}\right)\left(-\frac{p}{2}\right)-i \Omega_{2} v+i \Omega_{3}\left(\frac{\partial}{\partial z}-\frac{\partial}{\partial \bar{z}}\right) \phi, \\
& 2\left(\frac{\partial}{\partial z}-\frac{\partial}{\partial \bar{z}}\right)\left(\frac{\partial \phi}{\partial z} \frac{\partial \phi}{\partial \bar{z}}\right) \\
& \quad=\left(\frac{\partial}{\partial z}-\frac{\partial}{\partial \bar{z}}\right)\left(-\frac{p}{2}\right)-i \Omega_{1} v+i \Omega_{3}\left(\frac{\partial}{\partial z}+\frac{\partial}{\partial \bar{z}}\right) \phi,
\end{aligned}
$$

$$
\begin{aligned}
& \frac{\partial \phi}{\partial z} \frac{\partial v}{\partial \bar{z}}+\frac{\partial \phi}{\partial \bar{z}} \frac{\partial \phi}{\partial z} \\
& \quad=2 \nu \frac{\partial^{2} v}{\partial z \partial \bar{z}}-i \Omega_{1}\left(\frac{\partial \phi}{\partial z}-\frac{\partial \phi}{\partial \bar{z}}\right)+\Omega_{2}\left(\frac{\partial \phi}{\partial z}+\frac{\partial \phi}{\partial \bar{z}}\right) .
\end{aligned}
$$

The addition and subtraction of the first two equations in (23) give

$$
\begin{aligned}
& \frac{\partial}{\partial z}\left(\frac{\partial \phi}{\partial z} \frac{\partial \phi}{\partial \bar{z}}+\frac{p}{2}-i \Omega_{3} \phi\right)=-\frac{i}{4} v \bar{\Omega} \\
& \frac{\partial}{\partial \bar{z}}\left(\frac{\partial \phi}{\partial z} \frac{\partial \phi}{\partial \bar{z}}+\frac{p}{2}+i \Omega_{3} \phi\right)=\frac{i}{4} v \Omega
\end{aligned}
$$


where $\Omega=\Omega_{1}+i \Omega_{2}$ and $\bar{\Omega}=\Omega_{1}-i \Omega_{2}$ and as already noticed $\Omega_{1}, \Omega_{2}$, and $\Omega_{3}$ are assumed to be constants. The third equation becomes

$$
\frac{\partial \phi}{\partial z} \frac{\partial v}{\partial \bar{z}}+\frac{\partial \phi}{\partial \bar{z}} \frac{\partial v}{\partial z}=2 v \frac{\partial^{2} v}{\partial z \partial \bar{z}}-i \frac{\partial \phi}{\partial z} \Omega+i \frac{\partial \phi}{\partial \bar{z}} \bar{\Omega} .
$$

It is interesting to note that the Coriolis effect does not appear in the $\phi$ equation which is related to two-dimensional velocity. Its effect appears on the equations for $v$ and the pressure.

Now suppose, for simplicity, that one of $\Omega$ 's is nonzero such that $\Omega_{1}=\Omega_{2}=0$ (in other words, $\Omega=\bar{\Omega}=0$ ) and $\Omega_{3} \neq 0$. In this case, we have

$$
\frac{\partial}{\partial z}\left(\frac{\partial \phi}{\partial z} \frac{\partial \phi}{\partial \bar{z}}+\frac{p}{2}-i \Omega_{3} \phi\right)=0 .
$$

The solution would be

$$
p(z, \bar{z})=-\frac{1}{2} \frac{\partial \phi}{\partial z} \frac{\partial \phi}{\partial \bar{z}}+i \Omega_{3} \phi+p_{0}(\bar{z}) .
$$

However, we try to make it simpler by taking $p_{0}(\bar{z})=0$. Then, the special solution of this equation becomes

$$
p=-\frac{1}{2} \frac{\partial \phi}{\partial z} \frac{\partial \phi}{\partial \bar{z}}+i \Omega_{3} \phi .
$$

So, we have the Navier-Stokes equation in complex coordinates under the Coriolis force with only one nonvanishing component of $\Omega$ :

$$
\begin{aligned}
\frac{\partial^{2} \phi}{\partial z \partial \bar{z}} & =0 \\
\frac{\partial \phi}{\partial z} \frac{\partial v}{\partial \bar{z}}+\frac{\partial \phi}{\partial \bar{z}} \frac{\partial v}{\partial z} & =2 v \frac{\partial^{2} v}{\partial z \partial \bar{z}} \\
p & =-\frac{1}{2} \frac{\partial \phi}{\partial z} \frac{\partial \phi}{\partial \bar{z}}+i \Omega_{3} \phi .
\end{aligned}
$$

The first equation means that $\phi$ is a holomorphic function. In this case, $\phi$ can be written as $\phi=f(z) \pm \bar{f}(\bar{z})$. By choosing negative sign and putting this holomorphic function $\phi$ with $\phi=f-\bar{f}$ into the above equations, we get

$$
\begin{aligned}
p & =-\frac{\partial f}{\partial z} \frac{\partial \bar{f}}{\partial \bar{z}}+2 i \Omega_{3}(f-\bar{f}), \\
\frac{\partial f}{\partial z} \frac{\partial v}{\partial \bar{z}}+\frac{\partial \bar{f}}{\partial \bar{z}} \frac{\partial v}{\partial z} & =4 v \frac{\partial^{2} v}{\partial z \partial \bar{z}} .
\end{aligned}
$$

So, when only one of $\Omega$ 's, say $\Omega_{3}$, is nonvanishing, the Coriolis effect appears only in the pressure equation.

When $\Omega_{1}=\Omega_{2}=\Omega_{3}=0$, the solution in complex coordinates is given by [6]

$$
\begin{aligned}
& \phi=\frac{1}{2}\left(z^{2}+\bar{z}^{2}\right), \\
& v=\operatorname{erf}\left(\sqrt{\frac{1}{v}}\left(-\frac{i}{2}\right)(z-\bar{z})\right), \\
& p=-2|z|^{2} .
\end{aligned}
$$

When we consider two-dimensional flow where the velocities are given by $u_{1}=\partial \phi / \partial x, u_{2}=\partial \phi / \partial y$, and $u_{3}=$ $v=0$ and $\vec{\Omega}=\left(0,0, \Omega_{3}\right)$, we get the result similar to the case obtained when only one component of $\Omega$ is nonvanishing. In Figure 1, we plotted $\phi$ and velocity of (31) for a given value of the viscosity 100 when the Coriolis effect does not exist. We see that the velocity vanishes along the $x$-axis.

Now, let us consider the case where all three components of $\Omega$ 's are nonvanishing. When all components of $\Omega$ 's are not zero, the Navier-Stokes equations become

$$
\begin{aligned}
& \frac{\partial^{2} \phi}{\partial z \partial \bar{z}}=0, \\
& \frac{\partial}{\partial z}\left(\frac{\partial \phi}{\partial z} \frac{\partial \phi}{\partial \bar{z}}+\frac{p}{2}-i \Omega_{3} \phi\right)=-\frac{i}{4} v \bar{\Omega}, \\
& \frac{\partial}{\partial \bar{z}}\left(\frac{\partial \phi}{\partial z} \frac{\partial \phi}{\partial \bar{z}}+\frac{p}{2}+i \Omega_{3} \phi\right)=\frac{i}{4} v \Omega, \\
& \frac{\partial \phi}{\partial z} \frac{\partial v}{\partial \bar{z}}+\frac{\partial \phi}{\partial \bar{z}} \frac{\partial v}{\partial z}=2 \nu \frac{\partial^{2} v}{\partial z \partial \bar{z}}-i \frac{\partial \phi}{\partial z} \Omega+i \frac{\partial \phi}{\partial \bar{z}} \bar{\Omega} .
\end{aligned}
$$

To solve these equations, we first find the solution to the equation $\partial^{2} \phi / \partial z \partial \bar{z}=0$ that comes from the incompressible condition of the fluid. As a simple solution, we put $\phi$ as follows:

$$
\phi=z^{2}-\bar{z}^{2}
$$

Then, the above equations become

$$
\begin{aligned}
& \frac{\partial}{\partial z}\left(-4 z \bar{z}+\frac{1}{2} P-i \Omega_{3}\left(z^{2}-\bar{z}^{2}\right)\right)=-\frac{i}{4} v \bar{\Omega}, \\
& \frac{\partial}{\partial \bar{z}}\left(-4 z \bar{z}+\frac{1}{2} P+i \Omega_{3}\left(z^{2}-\bar{z}^{2}\right)\right)=\frac{i}{4} v \Omega .
\end{aligned}
$$

By differentiating with $\partial / \partial \bar{z}$ and $\partial / \partial z$ on the first and the second equations above, in turn we get equations like

$$
\begin{aligned}
& \frac{\partial^{2}}{\partial \bar{z} \partial z}\left(-4 z \bar{z}+\frac{1}{2} P-i \Omega_{3}\left(z^{2}-\bar{z}^{2}\right)\right)=-\frac{i}{4} \frac{\partial v}{\partial \bar{z}} \bar{\Omega}, \\
& \frac{\partial^{2}}{\partial z \partial \bar{z}}\left(-4 z \bar{z}+\frac{1}{2} P+i \Omega_{3}\left(z^{2}-\bar{z}^{2}\right)\right)=\frac{i}{4} \frac{\partial v}{\partial z} \Omega .
\end{aligned}
$$

As a result of further manipulation, we get the following equations:

$$
\begin{aligned}
-4+\frac{1}{2} \frac{\partial^{2} P}{\partial z \partial \bar{z}} & =-\frac{i}{4} \frac{\partial v}{\partial \bar{z}} \bar{\Omega} \\
\text { or }-4+\frac{1}{2} \frac{\partial^{2} P}{\partial z \partial \bar{z}} & =\frac{i}{4} \frac{\partial v}{\partial z} \Omega
\end{aligned}
$$

which turns out to be

$$
-\frac{\partial v}{\partial \bar{z}} \bar{\Omega}=\frac{\partial v}{\partial z} \Omega
$$




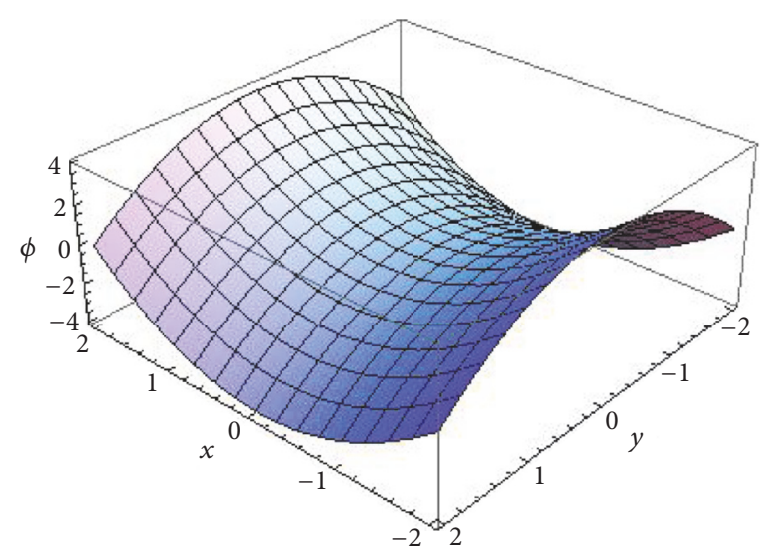

(a) $\phi$

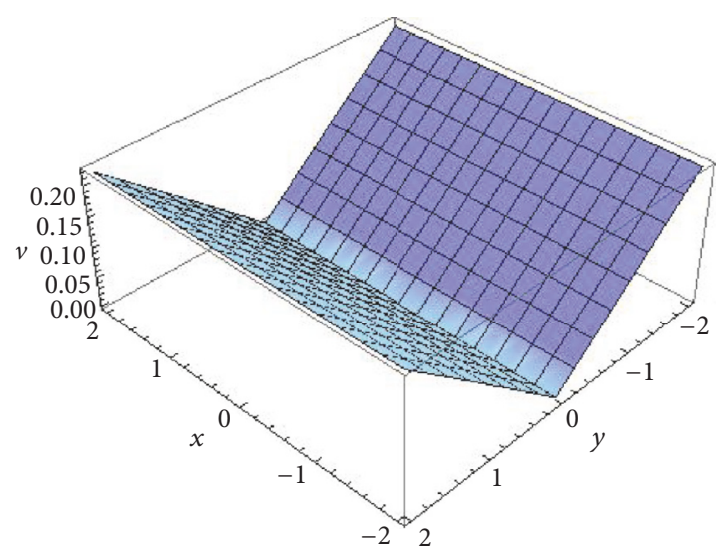

(b) $v$

FIGURE 1: Plots of profiles of absolute value of $\phi$ and velocity $v$ when $\Omega$ 's vanish.

The further application of partial derivatives gives the relation

$$
\begin{aligned}
\frac{\partial^{2} v}{\partial z \partial \bar{z}} \Omega & =-\frac{\partial^{2} v}{\partial \bar{z} \partial \bar{z}} \bar{\Omega}, \\
\text { or } \frac{\partial^{2} v}{\partial z \partial \bar{z}} \bar{\Omega} & =-\frac{\partial^{2} v}{\partial z \partial z} \Omega .
\end{aligned}
$$

The remaining equation

$$
\frac{\partial \phi}{\partial z} \frac{\partial v}{\partial \bar{z}}+\frac{\partial \phi}{\partial \bar{z}} \frac{\partial v}{\partial z}=2 v \frac{\partial^{2} v}{\partial z \partial \bar{z}}-i \frac{\partial \phi}{\partial z} \Omega+i \frac{\partial \phi}{\partial \bar{z}} \bar{\Omega}
$$

becomes

$$
2 z \frac{\partial v}{\partial \bar{z}}-2 \bar{z} \frac{\partial v}{\partial z}=2 v \frac{\partial^{2} v}{\partial z \partial \bar{z}}-2 i z \Omega-2 i \bar{z} \bar{\Omega} .
$$

With the help of $-(\partial v / \partial \bar{z}) \bar{\Omega}=(\partial v / \partial z) \Omega$, we have the secondorder differential equations. Let us now solve the secondorder partial differential equation:

$$
v \frac{\Omega}{\bar{\Omega}} \frac{\partial^{2} v}{\partial z^{2}}-\left(\frac{\Omega}{\bar{\Omega}} z+\bar{z}\right) \frac{\partial v}{\partial z}+i \Omega z+i \bar{\Omega} \bar{z}=0 .
$$

First, let us set $\partial v / \partial z=v_{z}$. Then, we have

$$
\frac{\partial v_{z}}{\partial z}-\frac{1}{v} \frac{\bar{\Omega}}{\Omega}\left(\frac{\Omega}{\bar{\Omega}} z+\bar{z}\right) v_{z}+\frac{1}{v} \frac{\bar{\Omega}}{\Omega}(i \Omega z+i \bar{\Omega} \bar{z})=0,
$$

or

$$
\frac{\partial v_{z}}{\partial z}-\frac{1}{v} \frac{\bar{\Omega}}{\Omega}\left(\frac{\Omega}{\bar{\Omega}} z+\bar{z}\right) v_{z}=-\frac{1}{v} \frac{\bar{\Omega}}{\Omega}(i \Omega z+i \bar{\Omega} \bar{z}) .
$$

Introducing the integrating factor $F$,

$$
\begin{aligned}
F & =e^{-\int d z(1 / v)(\bar{\Omega} / \Omega)((\Omega / \bar{\Omega}) z+\bar{z})} \\
& =e^{-(1 / v)\left(z^{2} / 2+(\bar{\Omega} / \Omega) \bar{z} z\right)+c_{1}(\bar{z})} \\
& =c_{2}(\bar{z}) e^{-(1 / v)\left(z^{2} / 2+(\bar{\Omega} / \Omega) \bar{z} z\right)},
\end{aligned}
$$

we have the solution to the differential equation

$$
\begin{aligned}
& F v_{z} \\
& \qquad=-c_{2}(\bar{z}) \frac{1}{\nu \Omega} i \int e^{-(1 / v)\left(z^{2} / 2+(\bar{\Omega} / \Omega) \bar{z} z\right)}\left(\frac{\Omega}{\bar{\Omega}} z+\bar{z}\right) d z .
\end{aligned}
$$

Introducing $\widetilde{z}=z+\bar{\Omega} \bar{z} / \Omega$, we get

$$
\begin{aligned}
F v_{z}= & -c_{2}(\bar{z}) \frac{1}{\nu \Omega} \\
& \cdot i\left[\frac{\Omega}{\bar{\Omega}} e^{(1 / 2 v)((\bar{\Omega} / \Omega) \bar{z})^{2}}(-\nu) e^{-(1 / 2 v) \tilde{z}^{2}}+c_{3}(\bar{z})\right] .
\end{aligned}
$$

By dividing by $F$ obtained above, we have the following $v_{z}:$

$$
v_{z}=\frac{i}{\bar{\Omega}}+\frac{c_{3}(\bar{z})}{c_{2}(\bar{z})} e^{(1 / 2 v)(z+(\bar{\Omega} / \Omega) \bar{z})^{2}-(1 / 2 v)((\bar{\Omega} / \Omega) \bar{z})^{2}} .
$$

Integrating further leads to the following:

$$
\begin{aligned}
v=\frac{i}{\bar{\Omega}} z+\frac{c_{3}(\bar{z})}{c_{2}(\bar{z})} e^{-(1 / 2 v)((\bar{\Omega} / \Omega) \bar{z})^{2}} \\
\cdot \frac{\sqrt{\pi} \operatorname{Erfi}[\sqrt{1 / 2 \nu}(z+(\bar{\Omega} / \Omega) \bar{z})]}{\sqrt{2 / \nu}},
\end{aligned}
$$

where the pressure $P$ can be determined by solving (36).

In Figure 2, we plotted $\phi$ and velocity $v$ for a nonvanishing value of the rotation. Compared to Figure 1, we see that the zero value axis rotates as $\Omega$ value increases. 


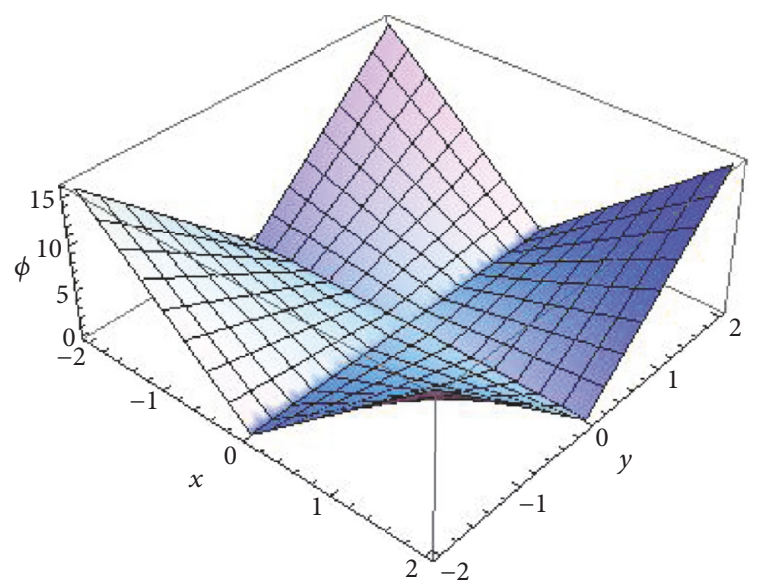

(a) $\phi$

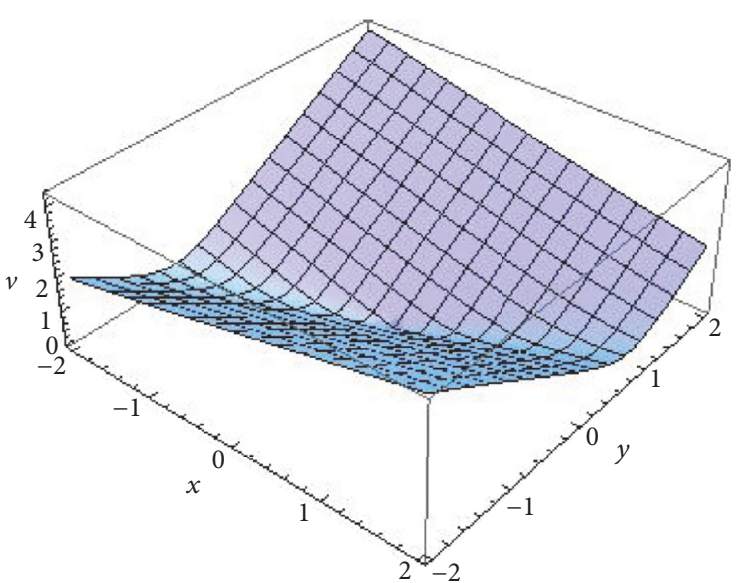

(b) $v(\Omega=1+i)$

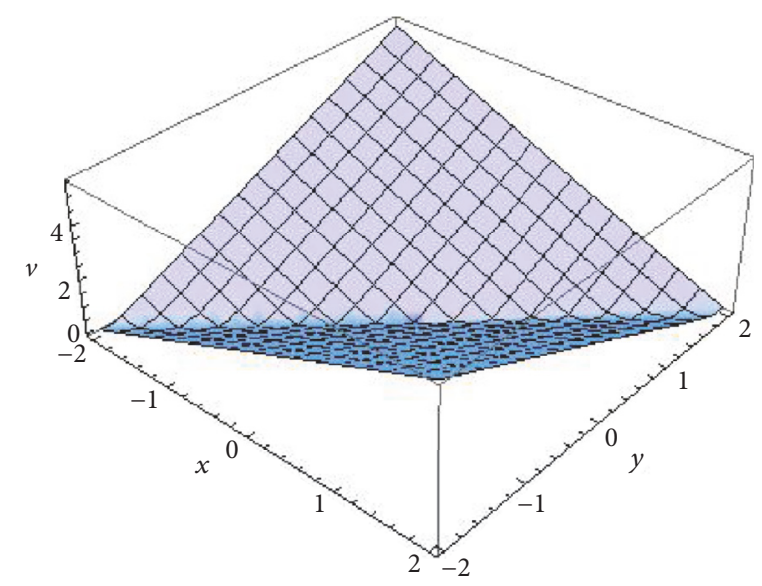

(c) $v(\Omega=10+i 10)$

FIGURE 2: Plots of profiles of absolute value of $\phi$ (a) and velocities $v$ when $\Omega=1+i$ (b) and $\Omega=10+i 10$ (c).

In summary, we have solved the Navier-Stokes equation with the assumption of $\phi$ in the presence of Coriolis force:

$$
\begin{aligned}
\phi= & z^{2}-\bar{z}^{2} \\
v= & \frac{i}{\bar{\Omega}} z+\frac{c_{3}(\bar{z})}{c_{2}(\bar{z})} e^{-(1 / 2 v)((\bar{\Omega} / \Omega) \bar{z})^{2}} \\
& \cdot \frac{\sqrt{\pi} \operatorname{Erfi}[\sqrt{1 / 2 \nu}(z+(\bar{\Omega} / \Omega) \bar{z})]}{\sqrt{2 / \nu}}, \\
P= & 8 z \bar{z}-\frac{i}{2} \bar{\Omega} \int v d z+P_{1}(z)+P_{2}(\bar{z}) .
\end{aligned}
$$

Now, let us consider the convective diffusion which is an application of the solution of Navier-Stokes equation. The equation of convective diffusion is given by

$$
\frac{\partial c}{\partial t}=D \nabla^{2} c-\vec{v} \cdot \vec{\nabla} c
$$

where $c$ is a concentration and $D$ is a diffusion coefficient. For two-dimensional flow where $\vec{v}=\left(v_{x}(x, y), v_{y}(x, y), 0\right)$ and assuming that $c(x, y)$ is independent of $z$ direction, the convective diffusion equation becomes

$$
D\left(\frac{\partial^{2} c}{\partial x^{2}}+\frac{\partial^{2} c}{\partial y^{2}}\right)=v_{x} \frac{\partial c}{\partial x}+v_{y} \frac{\partial c}{\partial y}
$$

which can be rewritten as

$$
\frac{\partial}{\partial x}\left(D \frac{\partial c}{\partial x}-\frac{\partial \phi}{\partial y} c\right)+\frac{\partial}{\partial y}\left(D \frac{\partial c}{\partial y}-\frac{\partial \phi}{\partial y} c\right)=0
$$

In this case, we assumed the potential flow in which the velocity is given by $(\partial \phi / \partial x, \partial \phi / \partial y, 0)$. Following the same procedure in [7], we get

$$
\begin{aligned}
c & =e^{-\phi / D}\left[\frac{1}{D} \int e^{-\phi / D} \frac{\partial \mathscr{C}}{\partial x} d x+f(y)\right] \\
& =e^{-\phi / D}\left[\frac{1}{D} \int e^{-\phi / D} \frac{\partial \mathscr{C}}{\partial y} d x+f(x)\right],
\end{aligned}
$$


where $\mathscr{C}$ is introduced such that

$$
\begin{aligned}
& D \frac{\partial c}{\partial x}-\frac{\partial \phi}{\partial x} c=-\frac{\partial \mathscr{C}}{\partial y} \\
& D \frac{\partial c}{\partial y}-\frac{\partial \phi}{\partial y} c=\frac{\partial \mathscr{C}}{\partial x}
\end{aligned}
$$

$\mathscr{C}$ satisfies Laplace's equation

$$
\frac{\partial^{2} \mathscr{C}}{\partial \xi^{2}}+\frac{\partial^{2} \mathscr{C}}{\partial \eta^{2}}=0,
$$

where we introduced $\xi$ and $\eta$ such that

$$
\begin{aligned}
& \xi(x, y)=\int e^{\phi / D} d x, \\
& \eta(x, y)=\int e^{\phi / D} d y .
\end{aligned}
$$

Solving the Navier-Stokes equation with the convective diffusion simultaneously, we can obtain the exact form of the concentration in our specific two-dimensional potential flow. If we choose $\phi=\left(z^{2}+\bar{z}^{2}\right) / 2=x^{2}-y^{2}$ and $\mathscr{C}=\xi+\eta$, then we have

$$
\begin{aligned}
c & =e^{-\left(x^{2}-y^{2}\right) / D}\left(\frac{x}{D}+f(y)+\text { constant }\right) \\
& =e^{-\left(x^{2}-y^{2}\right) / D}\left(\frac{y}{D}+g(x)+\text { constant }\right) .
\end{aligned}
$$

Here, $\xi$ and $\eta$ are given by

$$
\begin{aligned}
\xi & =\int e^{\left(x^{2}-y^{2}\right) / D} d x \\
& =e^{-y^{2} / D} \sqrt{D \pi} \frac{\operatorname{erfi}(x / \sqrt{D})}{2}+\text { const, } \\
\eta & =\int e^{\left(x^{2}-y^{2}\right) / D} d y=e^{x^{2} / D} \sqrt{D \pi} \frac{\operatorname{erf}(y / \sqrt{D})}{2}+\text { const. }
\end{aligned}
$$

In three dimensions, we see that the convective diffusion equation becomes

$$
\begin{aligned}
D \nabla^{2} c= & \vec{v} \cdot \vec{\nabla} c \\
\Longrightarrow & D\left(\frac{\partial^{2} c}{\partial x^{2}}+\frac{\partial^{2} c}{\partial y^{2}}+\frac{\partial^{2} c}{\partial z^{2}}\right) \\
= & v_{x} \frac{\partial c}{\partial x}+v_{y} \frac{\partial c}{\partial y}+\frac{\partial c}{\partial z}, \\
\Longrightarrow & \frac{\partial}{\partial x}\left(D \frac{\partial c}{\partial x}-v_{x} c\right)+\frac{\partial}{\partial y}\left(D \frac{\partial c}{\partial y}-v_{y} c\right) \\
& +\frac{\partial}{\partial z}\left(D \frac{\partial c}{\partial z}-v_{z} c\right)=0 .
\end{aligned}
$$

If we assume that the three-dimensional velocity is represented by $(\partial \phi(x, y) / \partial x, \partial \phi(x, y) / \partial y, v(x, y))$ and the last term vanishes, we can put

$$
D \frac{\partial c}{\partial z}-v_{z} c=g(x, y) \text { or constant. }
$$

In this case, we can solve the above equation by setting concentration as $c(x, y, z)=c(x, y) c(z)$. The solution of $c(z)$ can be obtained from the last equation and $c(x, y)$ can be obtained as was done above. In order to know the concentration $c(x, y, z)$ in this simplified example, we need to use solution (49). Here, we have

$$
\begin{gathered}
c(x, y, z)=c(z) c(x, y) \\
=\frac{D}{v(x, y)}\left(e^{z+z_{0}}-\frac{g(x, y)}{c(x, y) D}\right) \\
\cdot e^{-\left(z^{2}+\bar{z}^{2}\right) / D}\left(\frac{x}{D}+f(x)\right) .
\end{gathered}
$$

In Figure 3, we plotted the concentration for vanishing $\Omega$, $\Omega=1+i$, and $\Omega=10+i 10$, respectively. The concentration is high along one of the axes when the rotation is zero but reduces along that direction as the rotation is included. Our further study, however, shows that the concentration grows along the diagonal line as the absolute value of the rotation becomes large.

Using the curl operator, we are able to read the equation of vorticity. It is interesting to note that the above results show that there is no dependence on $\Omega_{3}$. The consideration of the independence on the third $(z)$ direction leads to the result of independence of that direction. The situation would be different if we take another expression of the velocity depending on the coordinates, say $(y, z)$. Let us suppose time independent and vanishing energy dissipation for the heat transfer in fluids. Then, we have the following equation:

$$
\vec{v} \cdot \vec{\nabla} T=\chi \nabla^{2} T
$$

where $\chi$ is thermal conductivity. Following the same procedure as was done for convective diffusion above, we will get the same formula for heat.

The solutions of vortices have a lot of applications in many areas. For example, let us consider the mixing of chemical species in a reactor. The molecular mixing and chemical reaction in the vortex pair were studied in [8]. The vortices enhance the diffusion rate and the reaction rate by increasing the interfacial area. In this case, the concentration depends on the vortex circulation $\Gamma$ which is defined as $\Gamma=\oint \vec{u}$. $\vec{d} \vec{l}=\int_{A} \vec{\omega} \cdot \vec{n} d A$ and Reynolds number $v$ called vortex strength $\Gamma / 2 \pi \nu$. From the constant shift of the vorticity vector under the constant Coriolis effect, we draw the result that the circulation reduces by the term coming from $-2 \Omega$. In [9], it was studied that vorticity accelerates the diffusion. It is closely related to the enhancement by the turbulence. It is interesting to find the relation between Coriolis effect and turbulence. We can study further these vorticity effects around the world. Those are about air of the atmosphere and the water at the sea. There are lots of chemical species and their reactions every moment including pollution as a kind of liquids or gases. The presence and the production of the vortices may help clean the environment by accelerating reactions. The solutions of vorticity and the solutions to the Navier-Stokes equation will give a better understanding of our nature. 


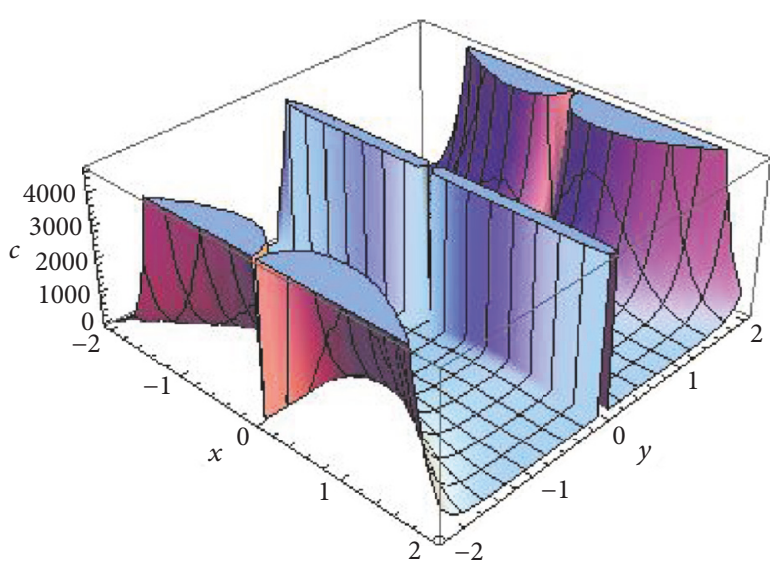

(a) $c(\Omega=0)$

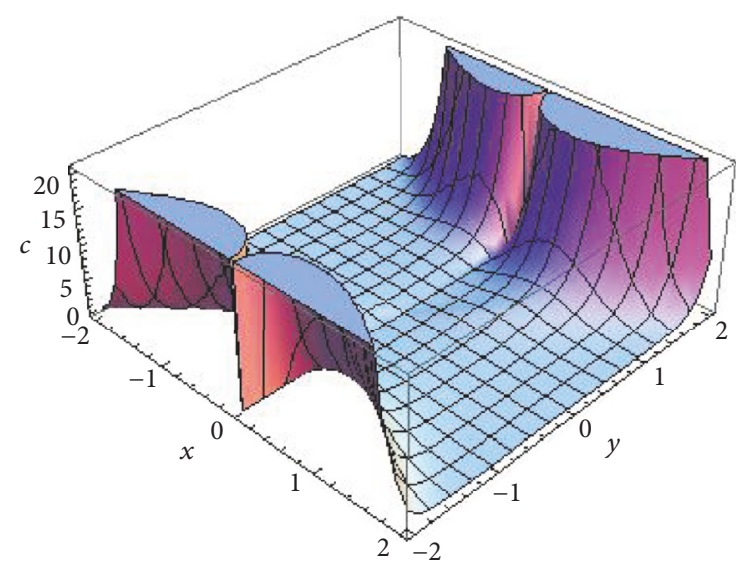

(b) $c(\Omega=1+i)$

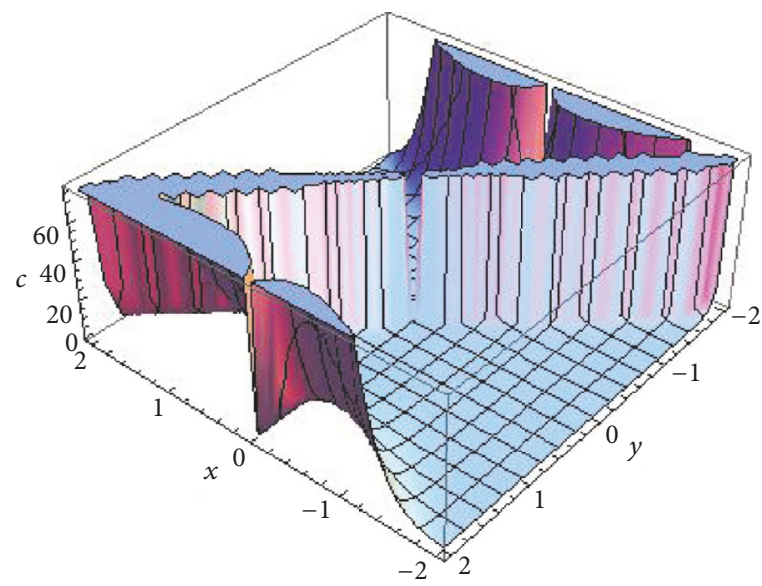

(c) $c(\Omega=10+i 10)$

Figure 3: Plots of profiles of absolute value of concentration $c$ when $\Omega=0$ (a), $\Omega=1+i$ (b), and $\Omega=10+i 10$ (c).

\section{Conclusions and Discussions}

The Navier-Stokes equations under the addition of the Coriolis force are discussed. The angular speed of the rotation is assumed to be a constant. We have seen that the vorticity can arise either for vortices depending on time only or for constant vortices as a result of Coriolis effect. It is interesting to note that, in two-dimensional flows, its effect on the vorticity vector $\omega$ results in a shift of the vorticity vector by $-2 \Omega$. It would be interesting to see what happens for the pair of vorticities or a number of vorticities.

By taking the specific expression for the velocity $u=$ $(\nabla \phi(x, y), v(x, y))$, we can solve exactly the nonlinear NavierStokes equations in the presence of Coriolis effect. The specific expression of the velocity leads to the secondorder partial differential equations of the velocity in two dimensions. With the independence of the velocity on the third direction, say $z$, we can analyze the velocity in two dimensions with complex coordinates.

Even though the Coriolis effect might be negligible in small scale, we may find some crucial role of its effect in the macroscopic regime. Particularly, to describe the air in the atmosphere, we need to include pressure and couple to temperature. The application of complex analysis such as conformal mapping (or transformation) and Joukowski transformation may help study more mathematically (by these transformations, many complicated flow boundaries may be transformed into regular flow boundaries and it is possible to obtain solutions for the flow around ellipses) $[6,10]$ and find a more general solution of the partial differential equations. If we can find the transformation between nonrotating and rotating frame, we may apply it to generate all known solutions easily.

The convective diffusion equation is solved for twodimensional potential flow. With the help of the solution of the Navier-Stokes equation, we get the velocity and find the concentration. The effect of the Coriolis rotation is absent in that case. Perhaps it can be deduced that this result is related to the chance of the rotation vector. The only possibility of the rotation vector is through the plane.

The application of the two-dimensional flows gives the exact motion of the air in our atmosphere and may predict the drifting particle motion that is harmful to creatures breathing air including humans. Moreover, the motion of the reactants inside a rotating reactor is also one of the applications yet with the coupling of temperature. 
In fluid (gas or liquid) motions related to the application to chemical reactions, the vorticity would be important. The behavior of the gas might affect the reactions. In [8], the effect of vortex pair was discussed in molecular mixing. In $[9,11]$, the enhancement of reaction by vortex was discussed. In [12], CFD method was applied to study the vortex effect on dispersion rate, pressure drop, and turbulent kinetic energy of mixing particles. It would be interesting to extend our work to these studies as well. In addition, it is interesting to study the stabilities of our work and other various flows under the Coriolis effect (see [13] for Couette flow with the relation to Taylor-Görtler vortices; they discussed that the Taylor-Görtler vortices are induced by destabilizing effect of accelerating Couette flow).

The understanding of the vortex and the solutions to the Navier-Stokes equation in various situations is very important. For instance, high Reynolds number turbulence is related to the vortex [14]. We can obtain the vortex from the solutions of Navier-Stokes equation. The multivortices solution is an interesting application to the reactions extending the effect of vortex pair. It might be necessary to use computers for the multivortices. It is also interesting to find the solution of Navier-Stokes equation giving multivortices.

\section{Conflicts of Interest}

The authors declare that they have no conflicts of interest.

\section{Acknowledgments}

This project is supported by the " $\mathrm{R} \& \mathrm{D}$ Center for Reduction of Non- $\mathrm{CO}_{2}$ Greenhouse Gases (RE2015001690003)" funded by the Korean Ministry of Environment (MOE) as "Global Top Environment R\&D Program.”

\section{References}

[1] A. R. Prasanna and B. Mukhopadhyay, "Effect of Coriolis Force on Accretion Flows around Rotating Compact Object," International Journal of Modern Physics D, vol. 12, p. 157, 2003.

[2] A. Korshunova, "Exact solutions to the compressible NavierStokes equations with the Coriolis and friction terms," http:// arxiv.org/abs/0807.4311.

[3] P. G. Saffman, Vortex Dynamics, Cambridge University Press, Cambridge, UK, 1992.

[4] E. B. Bogomolny, "Stability of classical solutions," Soviet Journal of Nuclear Physics, vol. 24, pp. 449-870, 1976.

[5] M. K. Prasad and C. M. Sommerfeld, "Exact Classical Solution for the 't Hooft Monopole and the Julia-Zee Dyon," Physical Review Letters, vol. 35, p. 760, 1975.

[6] M. Z. Bazant and H. K. Moffatt, "Exact solutions of the Navier-Stokes equations having steady vortex structures," Journal of Fluid Mechanics, vol. 541, no. 55, 2005.

[7] M. Sen and K. T. Yang, "Laplace's equation for convective scalar transport in potential flow," Proceedings of the Royal Society of London. Series A: Mathematical and Physical Sciences, vol. 456, p. 3041, 2004.
[8] B. M. Categen and J. P. Aguirre, "Analysis of molecular mixing and chemical reaction in a vortex pair," Physics of Fluids, vol. 2, no. 12, p. 2211, 1990.

[9] P. Flohr and J. C. Vassilicos, "Accelerated scalar dissipation in a vortex," Journal of Fluid Mechanics, vol. 348, pp. 295-317, 1997.

[10] M. Z. Bazant, "Conformal mapping of some non-harmonic functions in transport theory," Proceedings of the Royal Society A, vol. 460, p. 1433, 2004.

[11] J. P. Crimaldi, J. R. Cadwell, and J. B. Weiss, "Reaction enhancement of isolated scalars by vortex stirring," Physics of Fluids, vol. 20, no. 7, Article ID 073605, 2008.

[12] J. D. Chung, J. W. Kim, and Y. M. Park, "A study on vortex generators to improve the mixing rate in the dry sorbent injection process of the flue gas desulfurization system," Korean Journal of Chemical Engineering, vol. 27, no. 1, p. 83, 2010.

[13] Y. H. Kim and M. C. Kim, "Relative energy stability analysis on the onset of Taylor-Görtler vortices in impulsively accelerating Couette flow," Korean Journal of Chemical Engineering, vol. 31, no. 12, p. 2145, 2014.

[14] D. I. Pullin and P. G. Saffman, "Vortex dynamics in turbulence," Annual Review of Fluid Mechanics, vol. 30, pp. 31-51, 1998. 


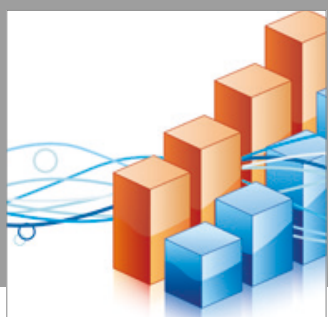

Advances in

Operations Research

vatersals

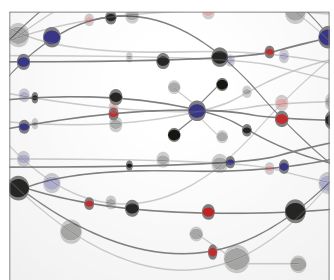

\section{The Scientific} World Journal
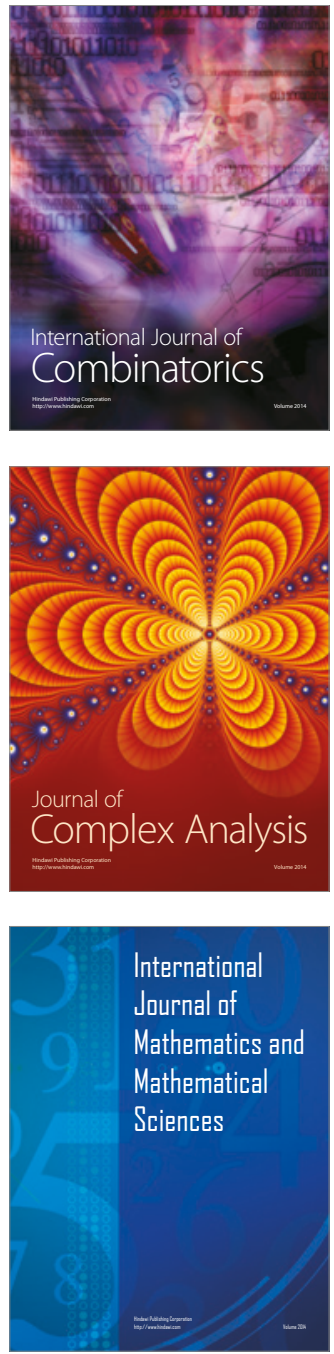
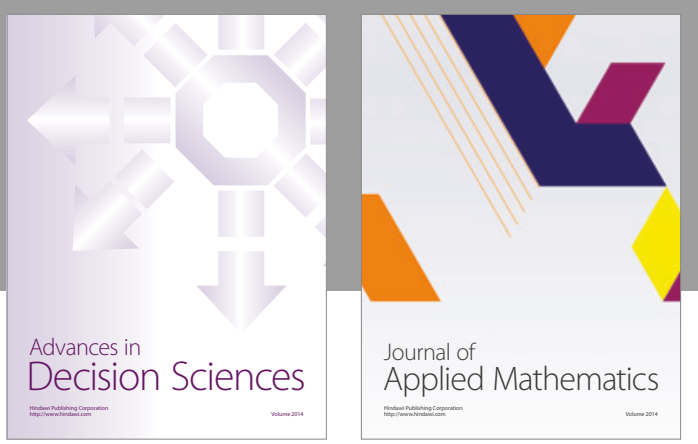

Algebra

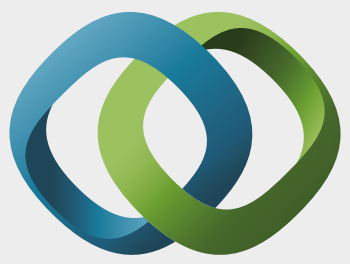

\section{Hindawi}

Submit your manuscripts at

https://www.hindawi.com
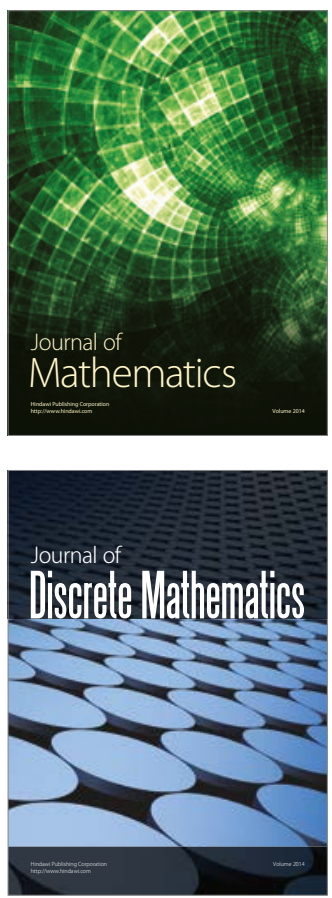

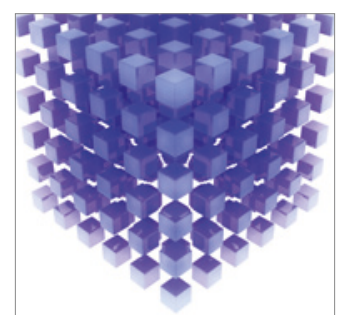

Mathematical Problems in Engineering
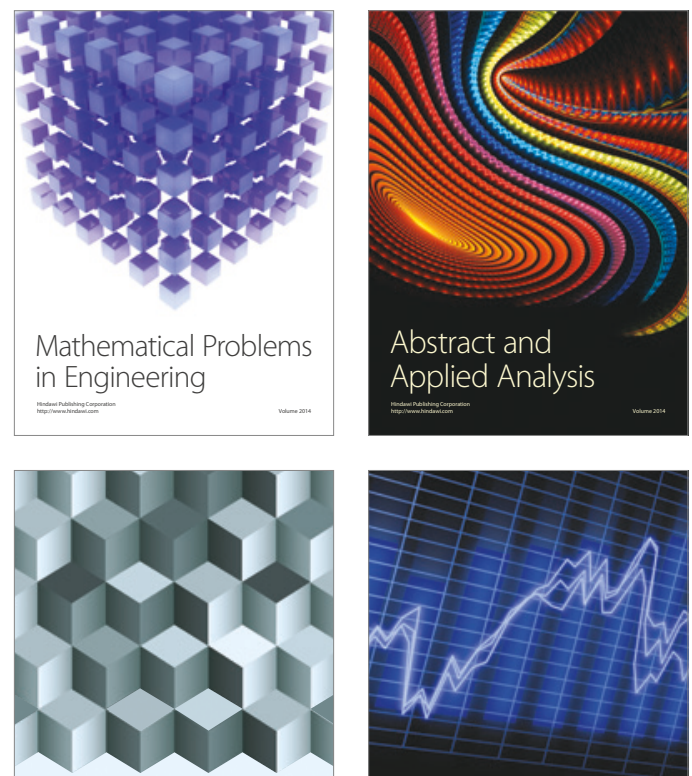

Journal of

Function Spaces

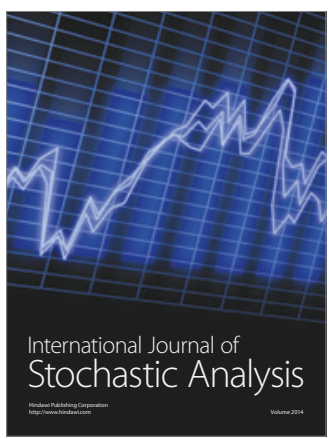

Probability and Statistics
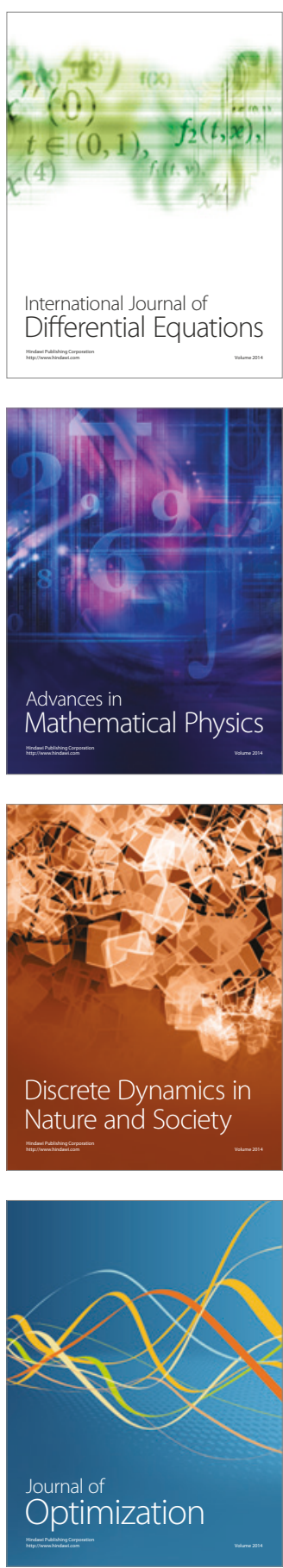